\title{
Cor triatriatum in a newborn
}

\author{
Davide Marini • Phalla Ou
}

Received: 29 January 2009 / Accepted: 2 February 2009/Published online: 10 March 2009

(C) Springer-Verlag 2009

A 1-month-old 3.1-kg infant was referred to our institution because of cyanosis and the suspicion of total anomalous pulmonary venous connection. The newborn underwent nongated cardiac CT (VCT LightSpeed, General Electric, Milwaukee, WI; slice thickness $0.625 \mathrm{~mm} \times 64$, rotation speed $0.4 \mathrm{~s}$, pitch $0.9,80 \mathrm{kVp}, \mathrm{mA}$ modulated during the acquisition) after peripheral injection of contrast agent ( $2 \mathrm{ml} / \mathrm{kg}$ of iohexol $300 \mathrm{mgI} / \mathrm{ml}$ injected at $0.5 \mathrm{ml} / \mathrm{s}$ ). CT elegantly revealed a fibromuscular membrane dividing the left atrium into two separate chambers (Fig. 1). The communicating fenestration was narrow and inferior (Fig. 2; arrow indicates the communicating fenestration). These images demonstrate the valuable diagnostic utility of high-resolution 3-D CT when cardiac US is not definitive, even for paediatric intracardiac abnormalities.

D. Marini $\cdot$ P. Ou $(\bowtie)$

Department of Paediatric Radiology,

Necker-Enfants Malades Hospital,

149 rue de Sèvres,

75743 Paris Cedex 15, France

e-mail: phalla.ou@nck.aphp.fr

D. Marini $\cdot$ P. Ou

Centre de Référence Malformations Cardiaques Congénitales

Complexes-M3C, Hôpital Necker-Enfants Malades,

Université Paris Descartes, AP-HP,

Paris, France

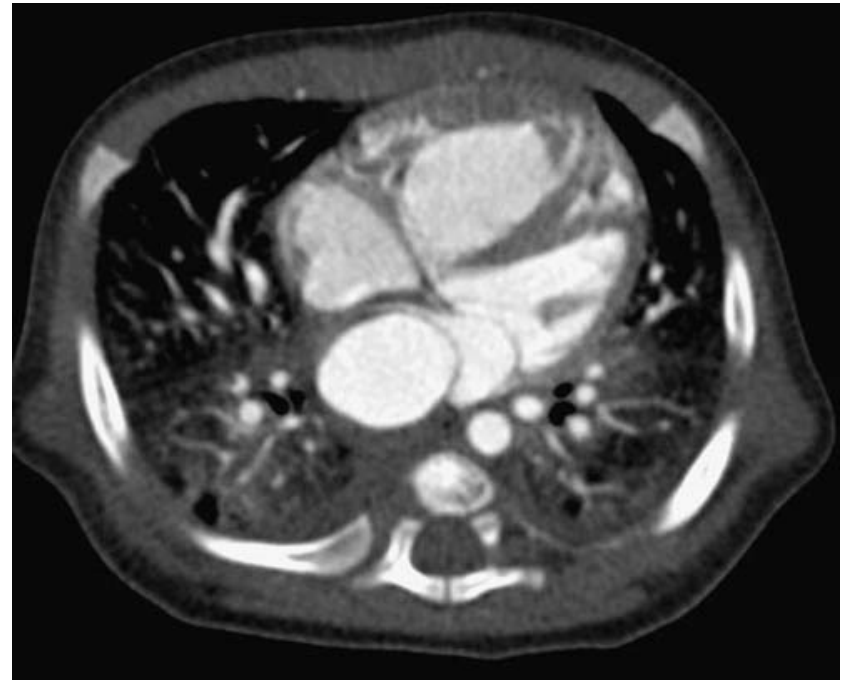

Fig. 1 Axial CT image

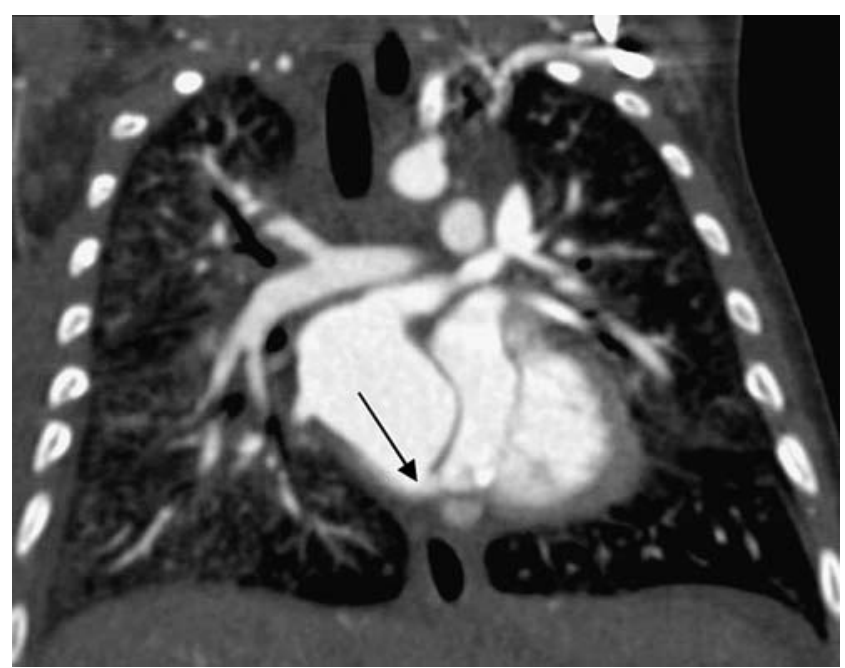

Fig. 2 Coronal CT image 